\title{
АДАПТАЦИЯ МЕТОДИКИ ОЦЕНКИ БАЗОВОГО АФФЕКТА М. ЙИК, ДЖ.А. РАССЕЛЛА И ДЖ.Х. ШТАЙГЕРА
}

\author{
М.Д. МАЛАШЕНКО ${ }^{a}$ Е.А. СТРИЖОВА
}

${ }^{a}$ Национальньй исследовательский университет Высшая школа экономики, 101000, Россия, Москва, ул. Мясницкая, д. 20

\section{Резюме}

В статье приводятся результаты апробации новой методики Оценки базового аффекта, построенной на основе 12-секторной круговой модели М. Йик, Дж.А. Расселла и Дж.Х. Штайгера (формат прилагательных). Данная методика позволяет оценить базовый аффект, который определяется авторами как элементарное эмоциональное переживание, лежащее в основе любой эмоции и представляющее собой сочетание двух факторов, ощущаемых человеком всегда: валентности (удовольствие - неудовольствие) и активности (активность - пассивность). Адаптация методики была проведена на выборке 200 человек. Для оценки надежности методики был вычислен коэффициент $\alpha$ Кронбаха для каждой шкалы. Надежность 11 из 12 шкал оказалась высокой или очень высокой, Результаты многомерного шкалирования показали, что адаптированная модель соответствует циркумплексу с двумя измерениями: «Активность» и «Удовольствие». Для оценки конструктной валидности был проведен регрессионный анализ, независимыми переменными которого были измерения базового аффекта, а зависимыми переменными выступали факторы двух других инструментов: Шкалы дифференциальных эмоций К. Изарда и методики Самооценки эмоциональных состояний А. Уэссмана и Д. Рикс. По итогам анализа удалось выявить, что фактор «Удовольствие» представлен в большем числе эмоций, нежели «Активность», тем не менее второй фактор имел больший вес в определенных эмоциях, в частности в «Интересе» и «Удивлении» Шкалы дифференциальных эмоций. Результаты регрессионного анализа соответствуют сущности эмоций, для которых были сделаны модели. Таким

Статья подготовлена в результате проведения работы № 15-01-0008 в рамках Программы «Научный фонд Национального исследовательского университета “Высшая школа экономики” (НИУ ВШЭ)» в 2015-2016 гг. и с использованием средств субсидии на государственную поддержку ведущих университетов Российской Федерации в целях повышения их конкурентоспособности среди ведущих мировых научно-образовательных центров, выделенной НИУ ВШЭ. 
образом, результаты психометрический проверки позволили сделать вывод о ее высокой внутренней надежности-согласованности и валидности. Практическая значимость методики состоит в возможности ее применения для оценки эмоционального состояния индивида, в том числе тогда, когда объект переживания неизвестен. Вероятно, методика позволяет более точно проводить кросс-культурные сравнения эмоциональных переживаний, поскольку меньше зависит от наименования эмоционального переживания в том или ином языке.

Ключевые слова: базовый аффект, эмоции, методики, метод, теория психологического конструирования эмоций.

\section{Введение}

Вопросы, связанные с оценкой эмоций, в настоящее время становятся более и более актуальными. Наряду с интеллектом был операционализирован концепт эмоционального интеллекта, важной темой стали определение и оценка роли эмоций в процессах мотивации, запоминания и обучения (Madan, 2013; Tulis, Fulmer, 2013), принятия решений (Lerner, Keltner, 2000), здоровья и самочувствия (Fredrickson, 2000) и др. Многие технологические компании работают над тем, чтобы создавать роботов, способных воспроизводить человеческие эмоции; появляются мобильные приложения, которые предназначены для того, чтобы делиться именно эмоциями. Представители таких профессий, как маркетолог, дизайнер или архитектор, в последнее время стали больше внимания уделять психологическому комфорту, что создало необходимость более четко разбираться в том, как возникают те или иные эмоции и как их можно вызвать.

С одной стороны, исследования в сфере эмоциональных переживаний позволяют определять причины возникновения эмоций, выделять их функции, классифицировать и изучать их взаимосвязь с различными психологическими и физиологическими процессами в организме. С другой стороны, эти открытия можно использовать для разработки специальных носимых устройств, позволяющих диагностировать эмоциональное состояние человека, усовершенствовать искусственный интеллект, разрабатывать дизайн объектов и пространств и т.п. Итак, изучение эмоций имеет важное значение для целого ряда областей: собственно психологии как науки и смежных областей: бизнес-практики, маркетинга, урбанистики и т.п.

\section{Проблемы в изучении эмоций}

Для изучения эмоциональных переживаний, в первую очередь, необходимо иметь возможность точно и правильно их оценивать. В данном контексте в психологической науке существует ряд проблем, нуждающихся в глубокой проработке.

Методологические проблемы. Первая проблема, связанная с исследованиями эмоциональной сферы, небольшое количество отечественных тестов, которые соответствуют международным стандартам (Батурин, 
Мельникова, 2009; Осин, 2012; Шмелев, 2004). На русском языке существует большой набор методик, оценивающих эмоции в контексте патологий, нервных расстройств, депрессии и прочих специфических условий, однако число инструментов для оценки эмоционального состояния любого индивида сравнительно невелико, многие из них созданы/адаптированы еще в XX в. Примерами таких методик являются САН («Самочувствие, активность, настроение»), методика Самооценки эмоциональных состояний (далее СЭС), Шкала дифференциальных эмоций К. Изарда (ШДЭ), методика оценки психическои активации, интереса, эмоционального тонуса, напряжения и комфортности и др. (Барканова, 2009), при этом данные об их психометрических показателях авторам найти не удалось. Недавно на русский язык была переведена методика PANAS (Осин, 2012), которая оценивает эмоциональное состояние человека с точки зрения позитивного либо негативного аффекта.

В англоязычной литературе, напротив, встречается большое разнообразие методик, оценивающих эмоциональные переживания разных уровней (подробный обзор см.: Mortillaro, Mehu, 2015): модель позитивных и негативных аффектов Дж. Уотсона и А. Теллегена (PANAS; Watson et al., 1988), существующая в нескольких вариантах, Pleasure-arousal-dominance (PAD), - модель разработанная Д.А. Расселлом и А. Мехрабианом на основе семантического дифференциала Ч. Осгуда (Меhrabian, Russell, 1974); существуют методики, определяющие эмоцио- нальное состояние человека по оценке рисунков и фотографий: например, методика Self-Assessment Маnikin (Bradley, Lang, 1994) или Методика оценки эмоционального отношения к продукту (The Product Emotion Measuring Instrument) (Desmet et al., 2007); для оценки текущего настроения разработан инструмент Краткая шкала рефлексии настроения (The Brief Mood Introspection Scale, BMIS; Mayer, Gaschke, 1988) и др.

\section{«Элементарность» или «комплексность»?}

Даже при условии расширения спектра методик, оценивающих эмоциональные состояния, необходимо понимать, какое явление оценивается. В психологическом дискурсе до сих пор не сформировалось консенсуса относительно того, что считается эмоцией, какие переживания можно считать элементарными, какие уровни эмоциональных переживаний в принципе существуют (Ильин, 2001; Izard, 2007).

Базовые эмоции. Ряд исследователей поддерживают идею существования базовых эмоций: биологически определенного набора переживаний, которые целостны и неделимы по своей сути. Так, Р. Плутчик выделяет восемь (Plutchik, 1991, 2002), К. Изард - десять (Izard, 1977), позже шесть (Izard, 2007), H.X. Фрийда - шесть (Frijda, 1986), М.Б. Арнольд - одиннадцать (Arnold, 1960), П. Экман, В.В. Фризен и П. Элсворт - шесть (Ekman et al., 1982), К. Отли и П.Н. Джонсон-Лэйрд пять (Oatley, Johnson-Laird, 1987), P.Е. Джек с соавт. - четыре (Jack et 
al., 2014), Дж. Панксепп - четыре базовые эмоции (Panksepp, 1982). Л. Аль-Шаваф с соавт. предлагают расширить список базовых эмоций Плутчика до всех переживаний, которые так или иначе участвуют в процессе приспосабливания организма к меняющейся ситуации (AlShawaf et al., 2015).

Теории, выделяющие базовые эмоции, можно условно разделить на два направления. Первое направление - психоэволюционные теории, берущие начало от идеи Дарвина о том, что эмоции формируются в процессе эволюции живых существ и являются врожденными приспособительными механизмами, направленными на самосохранение. Проявлением эмоций являются изменения в физиологических процессах организма и поведении. В «новой» эволюционной теории понятие и свойства базовой эмоции пересматриваются: базовая эмоция выделяется на основе не только жизненных процессов, связанных с самосохранением, но и деятельности и потребностей, связанных с репродукцией; при этом базовыми эмоциями считаются и те, которые характерны исключительно для человека, как, например, ревность (Ibid.).

Критика подобных теорий заключается в том, что зачастую в них больше исключений, чем правил (Barrett, 2006; Lindquist et al., 2013; Russell, 2003). Несмотря на то что имеется ряд признаков, которые считаются определяющими для разных эмоций, очень редко случается, что все они действуют одновременно. Как правило, исследователи объясняют это наличием специальных механизмов наподобие подавления.
Однако наличие большого количества таких механизмов и большая вариативность в проявлении одних и тех же эмоций в зависимости от ситуации ставит под сомнение состоятельность таких теорий и их объяснительную силу. Результаты и условия некоторых опытов по выделению базовых эмоций также подвергаются критике: например, тесты, в которых испытуемым предлагается определить эмоцию по выражению лица на картинке или по голосу, как правило, предлагают такой стимульный материал, в котором данные эмоции гипертрофированы, вследствие чего человеку действительно оказывается проще их определить, в то время как в жизни настолько сильные проявления эмоций встречаются редко, и, предположительно, категоризировать менее интенсивные эмоции человеку сложнее (Barrett, 2006).

Некоторые исследования приводят возможные доказательства существования базовых эмоций на нейрофизиологическом уровне (Öhman, 2005). Тем не менее, если эмоции на самом деле не являются целостными сущностями, очаги реакции в мозге человека могут характеризовать какой-то из составляющих элементов эмоции, а не всю эмоцию целиком. Для того чтобы исключить подобную возможность, необходимо проведение исследований, в которых одновременно оценивались бы разные проявления эмоций: нейрофизиологические реакции, изменение кровяного давления, мимики лица и др. Высокие корреляции между всеми этими переменными покажут, что у них имеется общая причина, которой и может являться базовая 
эмоция. При этом необходимо рассматривать данные проявления сразу для нескольких базовых эмоций, чтобы продемонстрировать, что сочетания реакций, характерные для одной эмоции, не могут быть свойственны другой.

Второе направление - оценочная теория, в рамках которой эмоции определяются не биологическими механизмами, а оценкой человеком ситуации. Все разнообразие базовых эмоций в таком случае определяется набором оценок, которым человек пользуется в разных ситуациях. Критика данного подхода заключается в том, что, хотя в нем и допускается большая вариативность эмоциональных реакций, все же его сторонники склонны выделять специальные паттерны оценок, определяющих конкретные базовые эмоции (Barrett, 2006).

Эмоции как комплексные образования. Наряду с теориями, постулирующими существование базовых эмоций как неких неделимых сущностей, существует также ряд теорий, представляющих эмоции или то, что принято обозначать эмоциями, как сложные явления, которые можно разложить на более простые элементы. Хотя разделение эмоций или эмоциональных переживаний на составляющие обладает рядом преимуществ, ученые-психологи не достигли консенсуса относительно необходимого и достаточного количества элементов, на которые эмоция/эмоциональное переживание должно быть разделено, и относительно самих этих элементов. Разные исследователи предлагают от двух (Russell, 1980; Yik et al., 2011), трех (Osgood et al., 1975; Russell,
Mehrabian, 1977), четырех (Fontaine et al., 2007; Veirman, Fontaine, 2015) до восьми измерений (Cochrane, 2009), при этом не всегда соглашаясь относительно того, что такое эмоция и какое эмоциональное переживание можно считать целостным.

\section{Теории психологического конструирования эмоций и концепция Дж.А. Расселла}

Базовый аффект как элементарное эмоциональное состояние. Важно отметить, что в случае с Дж.А. Расселлом, Л.Ф. Барретт, М. Йик и рядом других исследователей - сторонников теории психологического конструирования эмоций (psychological construction theory) - речь все же идет не об эмоции, а об элементарном эмоциональном переживании, которое называется «базовый аффект». Прочие измерения, предлагаемые другими исследователями как основные свойства эмоции, относятся в рамках данной логики не к самой эмоции, а к другим явлениям, происходящим параллельно с эмоциональным переживанием (Russell, 1980).

Названные выше исследователи ставят под вопрос естественность и универсальность феномена эмоции (Barrett, 2006; Russell, 1991, 2003), т.е. говорят, что они не могут быть выделены в психике человека как сущности с четкими границами, однозначно отделяющими их от других сущностей. Л.Ф. Барретт (Barrett, 2006) приводит множество аргументов против того, что базовая эмоция - некая неделимая сущность. При этом, ссылаясь на различных авторов, она также демонстрирует свидетельства 
того, что эти эмоции можно разделить на составляющие части. Некоторые психологи полагают, что эмоция является не естественным феноменом, а искусственным конструктом (Lindquist, Barrett, 2008; Russell, 2003). В соответствии с данной логикой эмоции считаются результатом категоризации, которая необходима человеку для упрощения социального взаимодействия и общения (Barrett, 2009).

В рамках теории психологического конструирования эмоций базовый аффект понимается как элементарное эмоциональное состояние, включающее два измерения: валентность (удовольствие - неудовольствие) $и$ активность (активность - пассивность).

Концепция Дж.А. Расселла (Ibid.) подразумевает, что человек постоянно находится в эмоциональном состоянии под названием «базовый аффект», определение которого было приведено выше. Помимо этого, он утверждает, что все объекты, окружающие нас, обладают аффективным качеством (affective quality) - способностью влиять на базовый аффект человека. При этом объект понимается в широком смысле и может представлять как материальный объект вне человека, так и некоторое событие, а также психические и физиологические процессы в самом человеке и т.п. Несмотря на то что любой объект обладает аффективным качеством, срабатывание или несрабатывание данного качества зависит от конкретной ситуации, направленности внимания человека и других факторов. По Расселлу, эмоции не являются объективно существующими в природе феноме- нами и четкое выделение и разделение эмоций типа ярости, страха, грусти представляет собой плод человеческой мысли, а не объективную реальность. Вместо слова «эмоция» Расселл употребляет словосочетание «эмоциональный эпизод» (emotional episode), понимая его как некое событие, в процессе которого конструируется то, что принято считать эмоцией. Эмоциональный эпизод складывается из базового аффекта, восприятия аффективного качества объекта, атрибуции, оценки ситуации, поведенческой реакции, определения собственных ощущений (emotional meta-experience) и эмоциональной регуляции. Данные элементы могут действовать в разной последовательности и комбинироваться в разных сочетаниях, причем не обязательно все они должны присутствовать в отдельном эмоциональном эпизоде. Назнообразием комбинаций и объясняется различие для одних и тех же эмоций, которые тем не менее проявляются по-разному, и вообще огромное разнообразие эмоциональных переживаний. К примеру, эпизод страха, когда мы смотрим фильм ужасов, отличается от эпизода страха при спасении от медведя, по крайней мере, тем, что в первом случае у нас иная оценка ситуации (фильм не угрожает нашей жизни) и другая поведенческая реакция - мы не убегаем, а сидим на месте.

В рамках идеи о базовом аффекте Расселл предложил круговую модель его оценки (Russell, 1980), которая подразумевала, что базовый аффект можно представить в виде точки на круге, разделенном на 8 секторов. Впоследствии данная модель 
была усовершенствована с увеличением количества секторов до 12, по 30 градусов, главные оси которого образованы параметрами «Валентность» (удовольствие - неудовольствие) и «Активность» (активность пассивность), а остальные 4 - промежуточные градации (Yik et al., 2011). Например, полуоси, составляющие северо-восточный квадрант, с востока на север определяют следующие параметры: «Активность», «Приятная активность», «Активное удовольствие», «Удовольствие» и т.д.

Результаты исследований подтверждают, что базовый аффект существует на нейрофизиологическом уровне (Posner et al., 2005; Wilson-Mendenhall et al., 2013).

Предположительно методика Дж.А. Расселла имеет ряд преимуществ перед более распространенной, но более простой моделью оценки позитивных и негативных аффектов Дж. Уотсона и А. Теллегена (PANAS; Watson et al., 1988), которая также подразумевает отход от базовых эмоций в пользу более элементарных измерений для эмоциональных переживаний. В первую очередь, модель с двумя измерениями более информативна, нежели модель с одним измерением: по сути, на основе PANAS можно лишь сказать, приятные или неприятные ощущения человек испытывает, и невозможно оценивать более специфические характеристики эмоционального переживания.

\section{Преимущества и критика теории психологического конструирования}

Теория психологического конструирования эмоций обладает неко- торыми преимуществами в сравнении с теориями, выделяющими базовые эмоции. В первую очередь, она позволяет сгладить различия, связанные с языковыми и культурными особенностями индивидов. У разных народов по-разному выстроен язык эмоций: не обязательно определенная категория, существующая в одном языке, присутствует в другом, а эмоциональные комплексы также могут состоять из разных элементов (Russell, 1991). Выделяя более элементарные измерения для эмоций, мы переходим к терминологии, которая более универсальна и, вероятно, позволяет более надежно проводить кросс-культурные сравнения. Помимо этого, за счет разделения эмоций на отдельные измерения данная теория дает возможность более эффективно оценивать варитивность эмоциональных переживаний, которые в рамках теорий, выделяющих базовые эмоции, были бы отнесены к одной и той же категории. Также с помощью данной теории можно рассматривать, оценивать и объяснять переживания, у которых нет выявленного источника.

К тому же эмоция, как правило, подразумевает определенный объект, эту эмоцию вызвавший. Базовый аффект как состояние, в котором человек находится всегда, позволяет рассматривать и объяснять с точки зрения эмоциональных переживаний ситуации, когда источника переживания либо нет вовсе, либо он трудно определим (например, необъяснимая тоска) (Russell, 2003).

Критика идеи о базовом аффекте. Некоторые ученые критикуют идею о базовом аффекте, аргументируя это тем, что за основу его выделения 
взяты слишком общие признаки. Из-за этого определенное сочетание двух измерений может быть характерно для двух и более различных по своей сути эмоциональных состояний: например, высокий уровень активности и низкий уровень удовольствия могут быть характерны как для ярости, так и для страха или обеспокоенности. Также некоторые исследователи утверждают, что две указанные шкалы не являются полностью независимыми, а именно это качество необходимо для разработки подобного рода измерений; при этом само по себе измерение активности, возможно, является слишком генерализованным и предположительно связано с любым другим измерением, на которое может быть разделена эмоция (Cochrane, 2009). К тому же сторонники концепций, связанных с базовыми эмоциями, утверждают, что если бы эмоция являлась искусственным конструктом, то чувствованию эмоции можно было бы обучить, чего существующей практикой пока не продемонстрировано; при этом проявления базовых эмоций встречаются и у четырехмесячных детей, которые еще не способны концептуализировать знание (Izard, 2007).

Комментируя идею о том, что у грудных детей тоже встречаются проявления эмоций, можно дать по крайней мере два возможных объяснения. Первое подразумевает, что базовые эмоции действительно врожденные, поэтому уже ребенок может их испытывать. Второе объяснение исходит из идеи о том, что эмоция все же состоит из ряда более простых элементов, которые могут либо участвовать в формировании эмоции, либо нет. С этой позиции эмоция, возникающая у ребенка, состоит только из тех элементов, которые доступны для его уровня психического и физиологического развития - т.е. связанных с непосредственным восприятием без участия когнитивных процессов. Относительно невозможности «научения» человека чувствовать эмоции также нельзя дать однозначного ответа, так как некоторые эмоциональные состояния социально обусловлены, как, например, стыд, и, по всей вероятности, во многом возникают благодаря рефлексии, приобретению человеком знаний и навыков общения. Что касается эмоций наподобие страха, то они, вероятно, более тесно связаны с инстинктами и биологической, нежели социальной, природой человека. В этом смысле научить человека бояться, чувствовать страх, наверное, невозможно можно лишь за счет знания и опыта расширить представления о том, чего можно бояться. Однако данный вывод не дает однозначного ответа на вопрос, составляет ли страх единую сущность или переживание страха определяется определенным набором факторов.

Вопрос о том, являются ли измерения активности и удовольствия независимыми, остается нерешенным. Некоторые исследования такой зависимости не обнаруживают (Anders et al., 2008), в то время как другие говорят о нестабильной связи активности и удовольствия, высокой вариативности, ситуационной и личностной обусловленности данной связи (Kuppens et al., 2013).

Аргумент о том, что в базовый аффект входят слишком общие изме- 
рения, можно принять в случае, если речь идет о взгляде на эмоции как на нечто целостное и неделимое. В свою очередь, сторонники рассматриваемого подхода говорят о том, что базовый аффект не тождествен эмоции, а является одним из ее необходимых элементов наряду с оценкой эмоции, регуляцией, поведенческой реакцией и т.п. и не может сам по себе объяснить различия в эмоциях (Lindquist et al., 2013; Russell, 2003).

Идея о том, что существуют разные уровни эмоциональных переживаний, высказана давно. Например, А.Н. Леонтьев выделяет аффекты, эмоции и чувства (Леонтьев, 1984). Они характеризуются разной продолжительностью и интенсивностью. Некоторые исследователи выделяют первичные, вторичные и третичные эмоции (Shaver et al., 2001). К. Изард выделяет собственно эмоции и эмоциональные схемы (Izard, 2007). Если мыслить в контексте разных уровней эмоциональных переживаний, то вполне логично, что нижние, более элементарные уровни должны включать меньшее количество измерений, чем более комплексные верхние. При этом измерения на базовом уровне должны быть как можно более общими и всеохватывающими.

Если следовать данной логике, теории, в которых эмоциональные переживания делятся на разное количество измерений, не обязательно противоречат друг другу - просто в одних приводятся измерения собственно эмоций (как, например, модель с 8 измерениями Т. Кочрейна), а в других - переживания более низкого или высокого порядка. Итак, аргумент насчет того, что пара- метр «активность» является «слишком» универсальным измерением, в данном контексте свидетельствует скорее в пользу, нежели против идеи его выделения как части базового эмоционального переживания.

Хотя идея о существовании базового аффекта и подвергается критике со стороны некоторых исследователей, все же есть весомые аргументы в пользу его существования, в частности, результаты нейрофизиологических исследований. Для некоторых аргументов, представляющих собой критику существования базового аффекта, пока что не найдено научных подтверждений, они существуют лишь в форме гипотез.

\section{Необходимость разработки новых методик и адаптации существующих}

В целом необходимость разработки новых или адаптации существующих методик для русского языка продиктована следующими причинами.

Добавление новых методик, с одной стороны, определяется требованиями современной психологической науки: развитием теорий эмоций и устареванием используемых методик. Те методики оценки эмоциональных состояний, которые имеются, были переведены довольно давно и, скорее всего, уже не соответствуют современному знанию об эмоциях. Например, ШДЭ с 10 эмоциями была разработана в конце 1970-х гг. (Izard, 1977) а спустя 30 лет К. Изард признал, что дифференциальных эмоций шесть (Izard, 2007). Шкалы для методики Самооценки эмоциональных состояний были разработаны 
около 50 лет назад (Wessman, Ricks, 1966). При этом данные по психометрическим характеристикам их адаптации, насколько известно автору, отсутствуют в открытых источниках. Естественно, эти методики за десятилетия могли быть пересмотрены частично или даже полностью, но ввиду недостаточного выбора адаптированных на русский язык методик по оценке эмоций приходится использовать устаревшие.

Новые методики требуются и бизнесу, так как в последнее время потребность в создании технологий, умеющих определять, воспроизводить или вызывать определенные эмоции в человеке, растет. В контексте настоящей статьи мы остановимся на причинах, по которым необходимо совершенствовать инструментарий, связанный с оценкой эмоциональных переживаний более элементарных, чем эмоция. Мы предполагаем, что при оценке элементарных эмоциональных переживаний можно найти такие связи с объектами, которые при оценке более сложных переживаний остаются незамеченными. Например, обстановка комнаты может не влиять на эмоции человека, но при этом все же оказывать воздействие на ощущения, связанные с чувством комфорта (Maslow, Mintz, 1956), или активность. Если мы будем оценивать эмоцию, эта связь, скорее всего, не будет обнаружена, так как есть слишком много воздействующих факторов помимо обстановки. Возможно, человек не всегда испытывает именно то, что считается эмоцией, а элементарные эмоциональные переживания, скорее всего, менее зависят от ситуации и больше связаны с вос- приятием внешних воздействий, нежели эмоции.

Добавление новой методики по оценке эмоциональных переживаний в пока что короткий список существующих на данный момент русскоязычных методик расширит диапазон выбора для психологов, исследующих эмоции, и обеспечит для них инструмент, который в определенных контекстах будет более эффективен, чем методики оценки эмоций, перечисленные выше.

Если удастся разработать инструмент, способный более точно улавливать воздействие внешних для человека объектов на психику, это даст стимул к развитию многих областей знания: архитектуры, ландшафтного проектирования, городского дизайна и др. Кроме того, это поможет вывести на новый уровень качество таких инструментов, как эмоциональные карты, т.е. более точно уловить влияние местности на переживания вне контекста ситуации, в которой находится конкретный человек.

\section{Методы}

Выборка. В исследовании приняли участие 200 человек в возрасте от 18 до 79 лет, из которых 139 (70\%) женщины и 61 (30\%) - мужчины. $65 \%$ респондентов (130 человек) имеют высшее образование.

Стимульный материал исследования был организован с помощью конструктора Google Формы. Исследование проводилось в июне-июле 2015 г. через Интернет посредством отправления испытуемым ссылки на указанный ресурс.

Описание инструмента. Методика позволяет оценить ситуативное 
эмоциональное состояние человека. По инструкции, респондент оценивает свое состояние в настоящий или прошлый момент времени.

Полная версия методики Оценки базового аффекта М. Йик, Дж.А. Расселла и Дж.Х. Штайгера (далее Методика оценки базового аффекта) состоит из трех тестов по 60 вопросов: 1) тест прилагательных, в котором репонденту предлагается ответить, насколько то или иное прилагательное описывает его психическое состояние, 2) тест «согласен - не согласен», в котором человеку предлагается в разной степени согласиться с представленным ему утверждением, связанным с эмоциями, 3) тест, в котором человек определяет, насколько то или иное описание совпадает с его текущим эмоциональным состоянием. Сокращенная версия подразумевает использование одного из трех форматов. В рамках данного исследования была произведена адаптация теста с прилагательными.

Суть методики, как понятно из названия, состоит в том, чтобы оценить базовый аффект человека по двум основным измерениям: «удовольствие» и «активность». Данные параметры могут быть представлены как в виде числа от -1 до 1, так и в виде точки на окружности, которая высчитывается путем взвешенного суммирования 60 ответов с умножением на коэффициенты, соответствующие их угловым позициям на круге. При оценке на круге базовый аффект, соответственно, определяется градусом и степенью его интенсивности.

Надежность шкал оригинальной методики в целом высокая ( $\alpha$ Крон- баха находится в пределах от 0.71 для фактора XII «Активность» до 0.93; Yik et al., 2001, p. 731), при этом методика оказалась валидной с точки зрения соответствия круговой модели и оценки эмоциональных переживаний, что было определено благодаря сравнению с аналогичными методиками (Ibid.).

Для валидизации данной методики были оценены корреляции переведенной методики с двумя другими: методикой Самооценки эмоциональных состояний (СЭС) и Шкалой дифференциальных эмоций К. Изарда (ШДЭ).

Тест Методика оценки базового аффекта состоит из 60 прилагательных, характеризующих текущее эмоциональное состояние человека. Их необходимо оценить по ранговой шкале от 1 (не соответствует) до 5 (полностью соответствует).

СЭС состоит из четырех вопросов, каждый из которых подразумевает выбор одного набора утверждений из десяти, представленных в виде ранговой шкалы от крайне негативного проявления того или иного состояния (1) до крайне позитивного проявления (10) (Барканова, 2009). Указанные утвержения относятся к текущему эмоциональному состоянию испытуемого. Информация по психометрическим показателям методики не приводится. Коэффициет $\alpha$ Кронбаха для СЭС, высчитанный авторами настоящей работы, оказался равным 0.802 (по сути тест представляет собой одну шкалу, состоящую из четырех измерений), что говорит об относительно высоком уровне надежности теста.

ШДЭ состоит из 30 прилагательных, оценивающих эмоциональное 
состояние человека в данный момент. Испытуемый должен отметить по ранговой шкале от 1 (совсем не подходит) до 4 (совершенно верно), насколько эти прилагательные соответствуют его текущему эмоциональному состоянию (Леонова, Кузнецова, 2009). Информация по психометрическим показателям методики не приводится. Сведения о надежности шкал методики на основе нашего исследования представлены в таблице 1.

Перевод методики на русский язык. Предварительно был составлен лонг-лист с несколькими вариантами перевода для каждого слова теста, после чего был сформирован итоговый набор прилагательных. Перевод теста был представлен группе профессиональных переводчиков на рецензию.

Стимульный материал состоял из вопросов трех методик с соответствующими инструкциями: переведенной версии Методики оценки базового аффекта (формат прилага- тельных), СЭС и ШДЭ. Перед указанными тестами испытуемым предлагалось оценить свое настроение по шкале от 1 (очень плохое) до 10 (очень хорошее).

\section{Результаты исследования}

Надежность-согласованность. Анализ внутренней надежностисогласованности для переведенной методики был проведен посредством вычисления коэффициента $\alpha$ Кронбаха для шкал, измеряемых в методике. В таблице 2 приведены результаты подсчетов коэффициента для 12 факторов оригинальной методики и ее адаптированного варианта.

Валидность. В качестве критериев валидности для перевода методики были выделены: а) расположение факторов модели по циркумплексу и б) оценка методикой элементарного эмоционального состояния.

Для подтверждения первого критерия использовалось многомерное шкалирование с использованием

Показатели надежности для ШДЭ

Таблица 1

\begin{tabular}{|c|c|}
\hline Фактор & $\alpha$ Кронбаха \\
\hline Ф1 - Интерес & 0.911 \\
\hline Ф2 - Радость & 0.903 \\
\hline Ф3 - Удивление & 0.917 \\
\hline Ф4 - Горе & 0.865 \\
\hline Ф5 - Гнев & 0.474 \\
\hline Ф6 - Отвращение & 0.841 \\
\hline Ф7 - Презрение & 0.807 \\
\hline Ф8-Страх & 0.737 \\
\hline Ф9 - Стыд & 0.866 \\
\hline Ф10 - Вина & 0.746 \\
\hline
\end{tabular}


Таблица 2

Оценка надежности шкал оригинальной методики Оценки базового аффекта и адаптированного варианта

\begin{tabular}{|c|c|c|}
\hline Фактор & $\begin{array}{c}\alpha \text { Кронбаха в } \\
\text { оригинальной } \\
\text { методике }\end{array}$ & $\begin{array}{c}\alpha \text { Кронбаха в } \\
\text { адаптированной } \\
\text { методике }\end{array}$ \\
\hline Ф1 - Приятная активность & 0.930 & 0.880 \\
\hline Ф2 - Активное удовольствие & 0.790 & 0.730 \\
\hline Ф3 - Удовольствие & 0.920 & 0.876 \\
\hline Ф4 - Дезактивированное удовольствие & 0.840 & 0.921 \\
\hline Ф5 - Приятная пассивность & 0.780 & 0.873 \\
\hline Ф6 - Пассивность & 0.770 & 0.739 \\
\hline Ф7 - Неприятная пассивность & 0.880 & 0.850 \\
\hline Ф8 - Пассивное неудовольствие & 0.930 & 0.895 \\
\hline Ф9 - Неудовольствие & 0.910 & 0.799 \\
\hline Ф10 - Активное неудовольствие & 0.920 & 0.923 \\
\hline Ф11 - Неприятная активность & 0.820 & 0.853 \\
\hline Ф12 - Активность & 0.710 & 0.653 \\
\hline Ф12-1* - Активность & - & 0.769 \\
\hline
\end{tabular}

* Без параметра «Глубоко чувствующий».

процедуры PREFSCAL на основе различий в пакете SPSS, результаты которого отражены на рисунке 1; показатель stress составил 0.130. В таблице 3 приводится матрица корреляций факторов модели между собой.

Для подтверждения второго критерия результаты переведенной методики были сопоставлены с результатами трех указанных выше инструментов. Их выбор был обусловлен тем, что они измеряют эмоциональные переживания вне зависимости от патологий, отклонений и других специфических обстоятельств и применимы для оценки ситуативных переживаний у любого психически здорового человека, как и адаптируемая методика.

Чтобы подтвердить, что адаптированная методика действительно оценивает текущее эмоциональное состояние человека, был проведен регрессионный анализ, в котором зависимыми переменными выступили 12 факторов методики оценки базового аффекта, независимыми переменными - шкалы перечисленных нами методик, включенных в батарею.

В таблице 4 можно увидеть результаты пошагового регрессионного анализа и анализа корреляции между измерениями базового аффекта и факторами других методик. 
Рисунок 1

Результаты многомерного шкалирования для 12 факторов Модели оценки базового аффекта

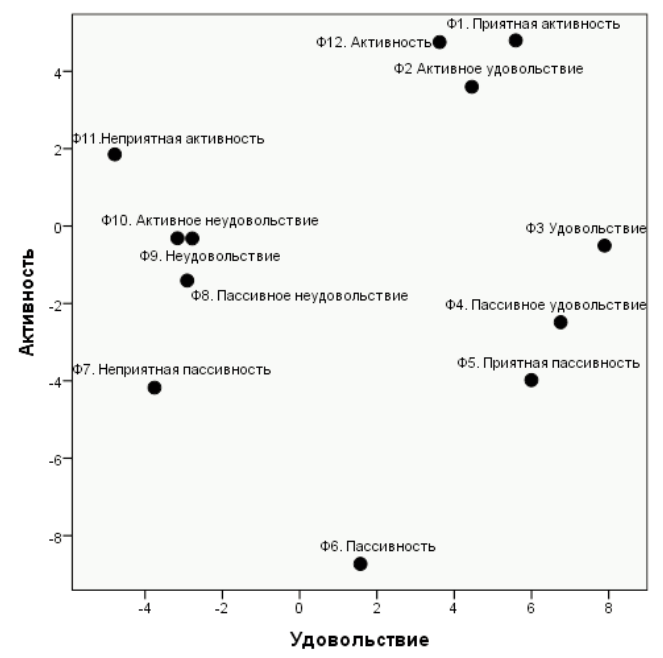

Таблица 3

Расчет корреляций для 12 факторов Методики оценки базового аффекта

\begin{tabular}{|c|c|c|c|c|c|c|c|c|c|c|c|}
\hline & $\Phi 1$ & Ф2 & Ф3 & $\Phi 4$ & Ф5 & $\Phi 6$ & Ф7 & Ф8 & Ф9 & $\Phi 10$ & $\Phi 11$ \\
\hline$\Phi 1$ & 1 & & & & & & & & & & \\
\hline$\Phi 2$ & $0.558^{* *}$ & 1 & & & & & & & & & \\
\hline Ф3 & $0.447^{* *}$ & $0.362^{* *}$ & 1 & & & & & & & & \\
\hline$\Phi 4$ & $0.354^{* *}$ & $0.273^{* *}$ & $0.508^{* *}$ & 1 & & & & & & & \\
\hline Ф5 & $0.191^{*}$ & 0.146 & $0.406^{* *}$ & $0.780^{* *}$ & 1 & & & & & & \\
\hline$\Phi 6$ & 0.030 & -0.082 & 0.134 & $0.347^{* *}$ & $0.525^{* *}$ & 1 & & & & & \\
\hline$\Phi 7$ & $-0.467^{* *}$ & -0.148 & $-0.380 * *$ & $-0.408^{* *}$ & $-0.254^{* *}$ & -0.053 & 1 & & & & \\
\hline Ф8 & $-0.320^{* *}$ & $-0.212^{*}$ & $-0.575^{* *}$ & $-0.494^{* *}$ & $-0.323^{* *}$ & -0.003 & $0.626^{* *}$ & 1 & & & \\
\hline Ф9 & $-0.188^{*}$ & -0.064 & $-0.548^{* *}$ & $-0.530^{* *}$ & $-0.409^{* *}$ & -0.131 & $0.577^{* *}$ & $0.771^{* *}$ & 1 & & \\
\hline Ф10 & -0.154 & -0.023 & $-0.472^{* *}$ & $-0.465^{* *}$ & $-0.383^{* *}$ & -0.127 & $0.588^{* *}$ & $0.737^{* *}$ & $0.853^{* *}$ & 1 & \\
\hline Ф11 & -0.057 & 0.003 & $-0.445^{* *}$ & $-0.533^{* *}$ & $-0.453^{* *}$ & -0.145 & $0.507^{* *}$ & $0.644^{* *}$ & $0.781^{* *}$ & $0.817^{* *}$ & 1 \\
\hline Ф12 & $0.565^{* *}$ & $0.579^{* *}$ & $0.230^{*}$ & $0.184^{*}$ & 0.008 & -0.092 & $-0.187^{*}$ & 0.017 & 0.115 & $0.202^{*}$ & $0.190^{*}$ \\
\hline
\end{tabular}

Примечание. Ф1 - Приятная активность, Ф2 - Активное удовольствие, Ф3 - Удовольствие, Ф4 - Дезактивированное удовольствие, Ф5 - Приятная пассивность, Ф6 - Пассивность, Ф7 Неприятная пассивность, Ф8 - Пассивное неудовольствие, Ф9 - Неудовольствие, Ф10 Активное неудовольствие, Ф11 - Неприятная активность, Ф12 - Активность.

${ }^{*} p<0.05,{ }^{* *} p<0.01$. 
Таблица 4

Характеристики регрессионных моделей, рассчитанных для подтверждения валидности, и корреляции измерений базового аффекта и факторов других рассмотренных методик

\begin{tabular}{|c|c|c|c|c|c|}
\hline & \multirow{2}{*}{$R^{2}$} & \multicolumn{2}{|c|}{$\beta$} & \multicolumn{2}{|c|}{$\begin{array}{c}\text { Коэффициенты } \\
\text { корреляции }\end{array}$} \\
\hline & & Активность & Удовольствие & Активность & Удовольствие \\
\hline \multicolumn{6}{|c|}{ Регрессионные модели для шкал методики ШдЭ } \\
\hline Итог ШДЭ & 0.514 & - & $0.717 * * *$ & 0.045 & $0.717^{* *}$ \\
\hline Радость & 0.516 & - & $0.718^{* * *}$ & 0.047 & $0.718 * * *$ \\
\hline Отвращение & 0.210 & - & $-0.458 * * *$ & 0.116 & $-0.458 * * *$ \\
\hline Интерес & 0.230 & $0.302 * * *$ & $0.405^{* * *}$ & $0.260^{* *}$ & $0.374^{* * *}$ \\
\hline Гope & 0.572 & - & $-0.756^{* * *}$ & 0.163 & $-0.756^{* * *}$ \\
\hline Гнев & 0.234 & $0.370 * * *$ & $-0.276^{* *}$ & $0.398 * * *$ & $-0.314^{* *}$ \\
\hline Страх & 0.226 & - & $-0.476^{* * *}$ & 0.149 & $-0.476^{* * *}$ \\
\hline Удивление & 0.139 & $0.347 * * *$ & $0.177^{*}$ & $0.328 * * *$ & 0.142 \\
\hline Стыд & 0.121 & - & $-0.348 * * *$ & 0.144 & $-0.348 * * *$ \\
\hline Презрение & 0.117 & - & $-0.341 * * *$ & 0.089 & $-0.341 * * *$ \\
\hline Вина & 0.195 & - & $-0.442 * * *$ & 0.067 & $-0.442 * * *$ \\
\hline \multicolumn{6}{|c|}{ Регрессионные модели для шкал методики СЭС } \\
\hline Итог СЭС & 0.737 & $0.163^{* *}$ & $0.860 * * *$ & 0.074 & $0.843^{* * *}$ \\
\hline $\begin{array}{l}\text { Уверенность в себе - } \\
\text { беспомощность }\end{array}$ & 0.371 & $0.231^{* *}$ & $0.588 * * *$ & 0.171 & $0.564^{* * *}$ \\
\hline $\begin{array}{l}\text { Энергичность - } \\
\text { усталость }\end{array}$ & 0.437 & $0.308^{* * *}$ & $0.617^{* * *}$ & $0.245^{* *}$ & $0.585^{* * *}$ \\
\hline $\begin{array}{l}\text { Приподнятость - } \\
\text { подавленность }\end{array}$ & 0.662 & $0.120^{*}$ & $0.817^{* * *}$ & 0.036 & $0.805^{* * *}$ \\
\hline $\begin{array}{l}\text { Спокойствие - } \\
\text { тревожность }\end{array}$ & 0.601 & $-0.142^{*}$ & $0.747 * * *$ & -0.219 & $0.762 * * *$ \\
\hline
\end{tabular}

${ }^{*} p<0.05,{ }^{* *} p<0.01,{ }^{* * *} p<0.001$.

\section{Обсуждение результатов}

Анализируя полученные данные, можно сделать вывод о том, что представленный тест обладает высокой степенью надежности и валидности.
Значение $\alpha$ Кронбаха для большинства шкал оказалось высоким (0.8-0.9 для 7 из 12 шкал, > 0.9 для двух шкал), что свидетельствует о высокой внутренней надежностисогласованности теста. Для одной 
шкалы наблюдается средний уровень надежности .653. Сравнительно более низкий уровень надежности для шкалы «Активность» может свидетельствовать о том, что набор прилагательных внутри данной шкалы не воспринимается или не полностью воспринимается как относящийся к единому параметру. Примечательно, что в оригинальной методике надежность этой шкалы также была сравнительно низкой (0.710 - Yik et al., 2011, p. 731). При исключении параметра «глубоко чувствующий» надежность шкалы увеличилась до 0.769, что позволяет говорить о том, что этот параметр должен быть либо полностью исключен из шкалы, либо для него требуется другой перевод.

Что касается валидности, на основе многомерного шкалирования было подтверждено, что 12 факторов модели оценки базового аффекта располагаются по кругу (рисунок 1). Как и в оригинальной методике, в результате многомерного шкалирования выделились два измерения, которые можно определить как «Активность - пассивность» и «Удовольствие - неудовольствие», причем ось «Активность - пассивность» оказывается немного смещенной в сторону полюса «Удовольствие» оси «Удовольствие - неудовольствие». Примечательно, что оси «Активность - пассивность» и «Удовольствие - неудовольствие» располагаются относительно друг друга под углом, близким к прямому, но не под прямым. При этом корреляционный анализ итоговых показателей активности и удовольствия выявил наличие незначимой корреляции между ними на уровне -.103 $(p=.273)$. Таким образом, наш анализ показывает, что «Удовольствие» и «Активность» с высокой степенью вероятности являются независимыми, а смещение факторов «Активность», «Пассивность», «Удовольствие» и «Неудовольствие» относительно друг друга может быть связано с недостаточно точным или полным набором прилагательных.

Угловое расположение факторов в целом соответствует оригинальной модели (рисунок 1): все факторы, которые связаны с удовольствием, имеют положительные значения по оси «Удовольствие», а все факторы, которые предполагают активность в противовес пассивности, имеют положительные значения по оси «Активность» двухмерного пространства, характеризующего базовый аффект. Отклонение от заданного теоретической моделью порядка демонстрируют только факторы «Активное удовольствие» и «Активное неудовольствие».

Есть несколько вариантов объяснения, почему два фактора модели находятся в позициях, смещенных относительно тех, которые предусмотрены исходной моделью. Во-первых, смещение фактора «Активное удовольствие» в сторону оси «Активность - пассивность», когда в исходной модели он должен располагаться ближе к оси «Удовольствие - неудовольствие», и фактора «Активное неудовольствие» к оси «Удовольствие - неудовольствие» (рисунок 2) могло наблюдаться из-за неточности перевода: могли быть подобраны такие слова, которые несут в себе больший вес активности, чем это должно быть в факторе «Активное удовольствие». К тому же 
Ориганальная модель оценки базового аффекта (Yik et al., 2011)

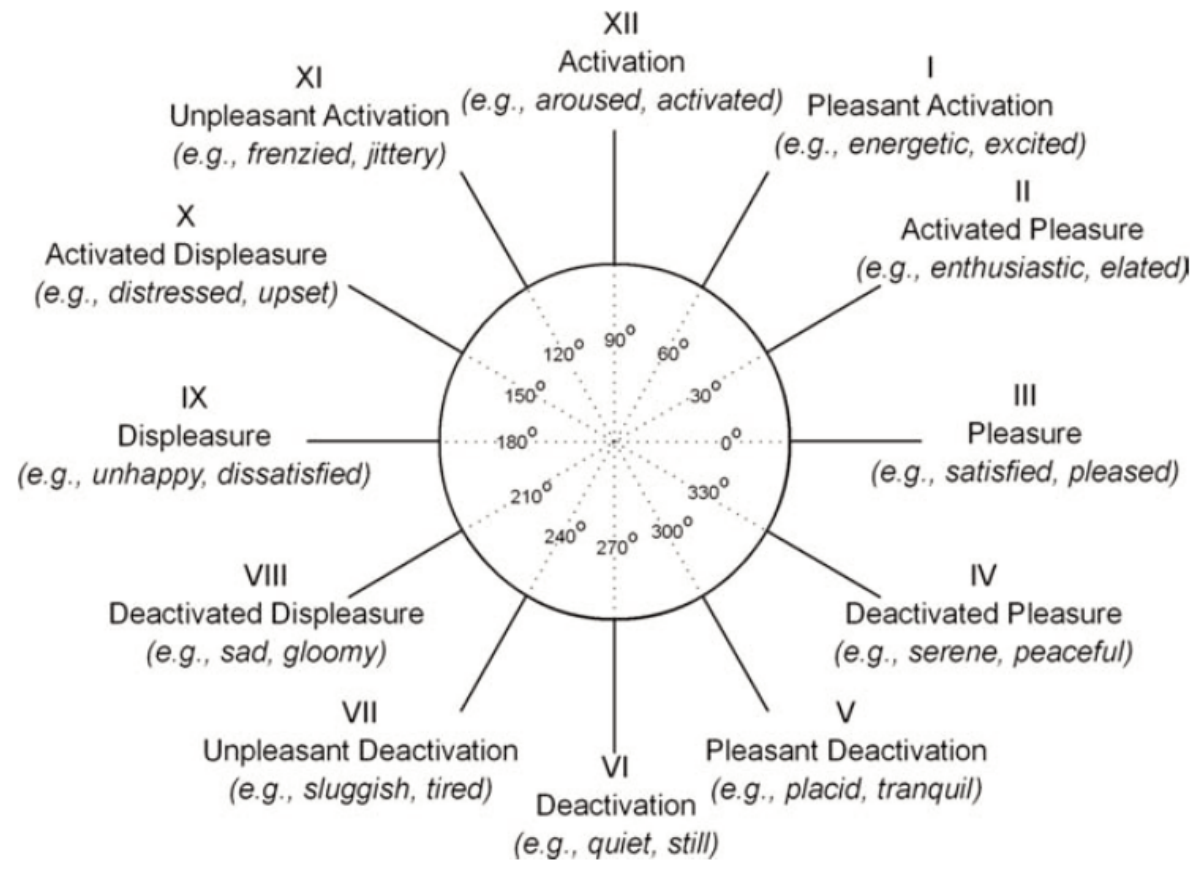

оригинальная методика подразумевает проведение 3 тестов по 60 вопросов с разными форматами ответов. За счет разных формулировок и большего количества проб, вероятно, различия между этими факторами оказывается проще определить и зафиксировать в формулировке самого вопроса. Кроме того, нами был выбран вариант, где испытуемому нужно оценить одно слово, a другие варианты подразумевают оценку выражений или словосочетаний, что позволяет передать некоторые смысловые оттенки более точно. Во-вторых, возможно, в некоторых случаях эмоциональное (в противовес смысловому) значение англоязычных прилагательных иногда ближе не к психологическому значению русского перевода, а к другим русскоязычным прилагательным. Иными словами, для англоязычной и русскоязычной выборок одно и то же слово может восприниматься и субъективно интерпретироваться по-разному, что, вероятно, продиктовано в том числе культурными различиями. Некоторые исследования показывают, что такие различия действительно имеют место (например: Markus, Kityama, 1991; Oyserman, Lee, 2008; Reddy, 2008).

Примечательно, что регрессионные модели, построенные для итоговых показателей других методик, обладают большей объяснительной силой, чем модели для их отдельных 
факторов: 74\% объясненной дисперсии для СЭС, $51 \%$ для ШДЭ и $12-23 \%$ для Презрения, Стыда, Горя, Гнева, Интереса, Отвращения, Удивления, Вины. Это может свидетельствовать о том, что базовый аффект действительно более тесно связан с общими, генерализованными эмоциональными переживаниями, нежели более специфическими, которые подпадают под понятие эмоции.

Разброс значений $R^{2}$ от 0.116 до 0.737 поддерживает логику теории психологического конструирования эмоций. Данная теория не утверждает, что базовый аффект определяет эмоцию. Напротив, помимо базового аффекта в формировании того, что мы называем эмоцией, в зависимости от ситуации участвуют еще ряд когнитивных процессов. Можно предположить, что те эмоции, для которых объясняется меньшая доля дисперсии, обладают более сложной природой и в их конструировании участвует большее количество элементов. Если рассматривать объяснительную силу моделей для конкретных эмоций, данная логика находит свое подтверждение: так, для горя и радости в методике ШДЭ регрессионная модель объясняет 57 и $52 \%$ дисперсии соответственно, а для сложных социально обусловленных эмоций наподобие презрения, стыда и вины, важную роль в формировании которых играет оценка ситуации, $R^{2}$ составляет лишь 12.12 и 20\% соответственно. При этом логика соотношения компонентов в регрессионных моделях соответствует здравому смыслу: например, интерес примерно в равной степени состоит из удовольствия и активности, так как суть данной эмо- ции заключается в повышении тонуса организма в поисках новой информации и получении удовлетворения от поиска. Гнев сопряжен с повышением активности и снижением уровня удовольствия. При этом здравому смыслу также соответствует низкая значимость активности для вины, стыда и презрения, где компонент удовольствия более сильный: когда человек испытывает стыд, он чувствует неудовольствие, но при этом уровень его активности, как правило, не меняется.

В целом параметр удовольствия обнаружил более высокий уровень и большее количество значимых корреляций с факторами из других тестов, нежели валентность. Кроме фактора «Напряжение» методики СЭС, не было ни одной регрессионной модели, в которой не было бы фактора «Удовольствие». Это противоречит аргументам Т. Кочрейна о том, что активность - универсальный параметр для всех эмоций (Cochrane, 2009).

Что касается суждения о том, что высокий уровень удовольствия не может сопровождаться низким уровнем активности, данное утверждение опровергается в настоящем исследовании: из 200 проб в 66 случаях разница между уровнем удовольствия и активностью составила больше 0.6 (значение каждого из измерений находится в интервале от -1 до 1), при этом в 8 случаях эта разница превысила 1. Подобное состояние можно характеризовать как приятную усталость.

Примечательно, что уровень активности среди всех испытуемых не привышал 0.429 (среднее значение -0.090 , стандартное отклонение - 
0.216 ; $\min --0.635$, $\max -0.429)$. Возможно, это обусловлено условиями тестирования: вероятность того, что человек, сидящий за компьютером, будет чувствовать повышенную активность, невелика. В то же время удовольствие человека меньше зависит от условий тестирования, поэтому здесь наблюдался больший разброс (среднее значение -0.246 ; стандартное отклонение -0.348 ; $\min -$ -0.953 , max -0.922$)$.

Знаки корреляций для негативных и позитивных эмоций в рассматриваемой методике практически полностью соответствуют логике и здравому смыслу: в случае с «Удовольствием» наблюдается отрицательная корреляция с факторами «Отвращение», «Горе», «Гнев», «Страх», «Стыд», «Презрение» и «Вина»; положительная корреляция для «Радости» и «Интереса»; нейтральная для удивления, поскольку оно не подразумевает определенной позитивно или негативно окрашенной реакции.

Значимых корреляций между полом и показателями «Удовольствия» и «Активности» обнаружено не было.

\section{Литература}

Барканова, О. В. (2009). Методики диагностики эмоциональной сферы: психологическиий практикум. Красноярск: Литера-принт.

Батурин, Н. А., Мельникова, Н. Н. (2009). Технология разработки тестов: часть І. Вестник Южно-Уральского государственного университета. Серия: Психология, 30(163), 4-14

Ильин, Е. П. (2001). Эмоции и чувства. СПб.: Питер.

Леонова, А. Л., Кузнецова, А. С. (2009). Психологические технологии управления состоянием человека. М.: Смысл.

Леонтьев, А. Н. (1984). Потребности, мотивы и эмоции. В кн. В. К. Вилюнас, Ю. Б. Гиппенрейтер (ред.), Психология эмоций. Тексты (с. 162-172). М.: Изд-во Московского университета.

Осин, Е. Н. (2012). Измерение позитивных и негативных эмоций: разработка русскоязычного аналога методики PANAS. Психология. Журнал Высшей школы экономики, 9(4), 91-110.

Шмелев, А. Г. (2004). Тест как оружие. Психология. Журнал Высшей школь экономики, 1(2), 40-53.

Ссылки на зарубежные источники см. в разделе References после англоязыного блока.

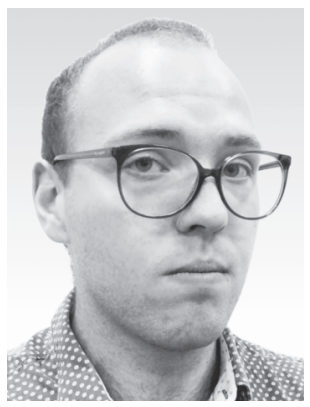

Малашенко Михаил Дмитриевич - выпускник, Высшая школа урбанистики им. А.А. Высоковского, Национальный исследовательский университет Высшая школа экономики.

E-mail: mihail_malashenko@mail.ru 


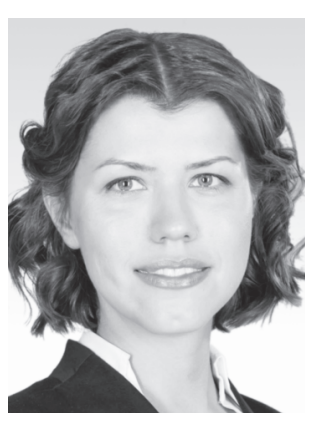

Стрижова Екатерина Андреевна - доцент, кафедра психоанализа и бизнес-консультирования, департамент психологии, факультет социальных наук, Национальный исследовательский университет Высшая школа экономики, кандидат психологических наук. Контакты: estrizhova@hse.ru

\title{
Adaptation of an Assessment Instrument for Core Affect by M. Yik, J.A. Russell and J.H. Steiger
}

\author{
Mikhail D. Malashenko ${ }^{\text {, }}$ Ekaterina A. Strizhova ${ }^{a}$ \\ ${ }^{a}$ National Research University Higher School of Economics, 20 Myasnitskaya Str., Moscow, 101000, \\ Russian Federation
}

\begin{abstract}
The article presents results of the validation of a novel method for assessment of an emotional state called core affect - 12-Point Affect Circumplex Scales, Adjective Format, developed by M. Yik, J.A. Russell and J.H. Steiger. This methodology allows the assessment of core affect - an elementary emotion state which lies in the core of every emotion and represents an integral unit consisting of two factors which every person feels permanently - valence (pleasure-displeasure) and arousal (activation-deactivation). The adaptation of the test was conducted on a sample of 200 individuals. In order to evaluate the construct validity, regression analysis was performed with the factors of two other tests as dependent variables: C. Izard's Differential Emotion Scale and Self-assessment of the Emotional State by A. Wessman and D. Ricks. Cronbach's alpha for each scale was estimated. Out of 12 , the reliability of 11 scales turned out to be either high or very high. The results of multidimensional unfolding have demonstrated that the adapted model fits a circumplex with two main axes, namely "Arousal" and "Pleasure". Regression analysis, in which dependent variables were the factors of two other instruments and the independent ones were the two dimensions of core affect, showed that "Pleasure" was present in a larger number of emotions than "Arousal". Nevertheless, "Arousal" was dominant in "Interest" and "Surprise" of the Differential Emotion Scale. The results of the regression analysis are commensurate with the essence of emotions it was conducted for. The results of the psychometric check of the instrument enable the conclusion of high levels of reliability and construct validity. Practical importance of the instrument is comprised by its applicability for the assessment of human's emotional state even when the object of the feeling is not obvious. The instrument is likely to be more accurate in conducting cross-cultural comparisons, as it is less dependent on different emotional concepts across different languages.
\end{abstract}

Keywords: core affect, emotion, instrument, method, psychological construction theory. 


\section{References}

Al-Shawaf, L., Conroy-Beam, D., Asao, K., \& Buss, D. M. (2015). Human emotions: An evolutionary psychological perspective. Emotion Review. Advance online publication. doi:10.1177/ 1754073914565518

Anders, S., Eippert, F., Weiskopf, N., \& Veit, R. (2008). The human amygdala is sensitive to the valence of pictures and sounds irrespective of arousal: An fMRI study. Social Cognitive and Affective Neuroscience, 3, 233-243. doi:10.1093/scan/nsn017

Arnold, M. B. (1960). Emotion and personality. New York: Columbia University Press.

Barkanova, O. V. (2009). Metodiki diagnostiki emotsional'noj sfery: psikhologicheskij praktikum [Instruments for diagnostics of emotional sphere: Psychological practicum]. Krasnoyarsk: Litera-print.

Barrett, L. F. (2006). Are emotions natural kinds? Perspectives on Psychological Science, 1, 28-58. doi:10.1111/j.1745-6916.2006.00003.x

Barrett, L. F. (2009). Variety is the spice of life: A psychological construction approach to understanding variability in emotion. Cognition and Emotion, 23(7), 1284-1306. doi:10.1080/ 02699930902985894

Baturin, N. A., \& Melnikova, N. N. (2009). Tekhnologiya razrabotki testov: chast' I [The technology of test development: Part I]. Bulletin of the South Ural State University. Series "Psychology", 30(163), 4-14.

Bradley, M. M., \& Lang, P. J. (1994). Measuring emotion: The self-assessment manikin and the semantic differential. Journal of Behavior Therapy and Experimental Psychiatry, 25(1), 49-59. doi:10.1016/0005-7916(94)90063-9

Cochrane, T. (2009). Eight dimensions for the emotions. Social Science Information, 48(3), 379-420. doi:10.1177/0539018409106198

Desmet, P. M. A., Porcelijn, R., \& van Dijk, M. (2007). Emotional design; application of a research based design approach. Journal of Knowledge, Technology and Policy, 20(3), 141-155.

Ekman, P., Friesen, W. V., \& Ellsworth, P. (1982). What emotion categories or dimensions can observers judge from facial behavior? In P. Ekman (Ed.), Emotion in the human face (pp. 39-55). New York: Cambridge University Press.

Fontaine, J. R. J., Scherer, K. R., Roesch, E. B., \& Ellsworth, P. C. (2007). The world of emotions is not two-dimensional. Psychological Science, 18(12), 1050-1057. doi:10.1111/j.1467-9280.2007.02024.x

Fredrickson, B. L. (2000). Cultivating positive emotions to optimize health and well-being. Prevention and Treatment, 3(1), 1-25. doi:10.1037/1522-3736.3.1.31a

Frijda, N. H. (1986). The emotions. New York: Cambridge University Press.

Il'in, E. P. (2001). Emotsii i chuvstva [Emotions and feelings]. Saint Petersburg: Piter.

Izard, C. E. (1977). Human emotions. New York: Plenum Press.

Izard, C. E. (2007). Basic emotions, natural kinds, emotion schemas, and a new paradigm. Perspectives on Psychological Science, 2, 260-280.

Jack, R. E., Garrod, O. G. B., \& Schyns, P. G. (2014). Dynamic facial expressions of emotion transmit an evolving hierarchy of signals over time. Current Biology, 24(2), 187-192. doi:10.1016/ j.cub.2013.11.064

Kuppens, P., Tuerlinckx, F., Russell, J. A., \& Barrett, L. F. (2013). The relation between valence and arousal in subjective experience. Psychological Bulletin, 139(4), 917-940. doi:10.1037/a0030811

Leonova, A. L., \& Kuznetsova, A. S. (2009). Psikhologicheskie tekhnologii upravleniya sostoyaniem cheloveka [Psychological technologies of management of human state]. Moscow: Smysl. 
Leontiev, A. N. (1984). Potrebnosti, motivy i emotsii [Needs, motives and emotions]. In V. K. Vilyunas \& Yu. B. Gippenreiter (Eds.), Psikhologiya emotsii. Teksty [The psychology of emotions. Texts] (pp. 162-172). Moscow: Moscow University Press.

Lerner, J. S., \& Keltner, D. (2000). Beyond valence: Toward a model of emotion-specific influences on judgement and choice. Cognition and Emotion, 14(4), 473-493.

Lewis, M. D. (2005). Bridging emotion theory and neurobiology through dynamic systems modeling. Behavioral and Brain Sciences, 28(2), 169-194. doi:10.1017/S0140525X0500004X

Lindquist, K. A., \& Barrett, L. F. (2008). Constructing emotion: The experience of fear as a conceptual act. Psychological Science, 19(9), 898-903. doi:10.1111/j.1467-9280.2008.02174.x

Lindquist, K. A., Siegel, E. H., Quigley, K. S., \& Barrett, L. F. (2013). The hundred-year emotion war: Are emotions natural kinds or psychological constructions? Comment on Lench, Flores, and Bench (2011). Psychological Bulletin, 139, 255-263. doi:10.1037/a0029038

Madan, C. R. (2013). Toward a common theory for learning from reward, affect, and motivation: The SIMON framework. Frontiers in Systems Neuroscience, 7, 59. doi:10.3389/fnsys.2013.00059

Markus, H., \& Kitayama, S. (1991). Culture and the self: Implications for cognition, emotion, and motivation. Psychological Reviere, 98, 224-253.

Maslow, A. H., \& Mintz, N. L. (1956). Effects of esthetic surroundings: I. Initial effects of three esthetic conditions upon perceiving "energy" and "well-being" in faces. The Journal of Psychology: Interdisciplinary and Applied, 41(2), 247-254. doi:10.1080/00223980.1956.9713000

Mayer, J. D., \& Gaschke, Y. N. (1988). The experience and meta-experience of mood. Journal of Personality and Social Psychology, 55(1), 102-111.

Mehrabian, A., \& Russell, J. A. (1974). An approach to environmental psychology. Cambridge, MA: MIT Press.

Mortillaro, M., \& Mehu, M. (2015). Emotions: Methods of assessment. In J. D. Wright (Ed.), International encyclopedia of the social and behavioral sciences (Vol. 7, pp. 519-525). Oxford, UK: Elsevier. doi:10.1016/B978-0-08-097086-8.25058-7

Oatley, K., \& Johnson-Laird, P. N. (1987). Towards a cognitive theory of emotions. Cognition and Emotion, 1, 29-50.

Öhman, A. (2005). The role of the amygdala in human fear: Automatic detection of threat. Psychoneuroendocrinology, 30(10), 953-958. doi:10.1016/j.psyneuen.2005.03.019

Osgood, C. E., May, W. H., \& Miron, M. S. (1975). Cross-cultural universals in affective meaning. Urbana, IL: University of Illinois Press.

Osin, E. N. (2012). Measuring positive and negative affect: development of a Russian-language analogue of PANAS. Psychology. Journal of Higher School of Economics, 9(4), 91-110. (in Russian)

Oyserman, D., \& Lee, S. W. S. (2008). Does culture influence what and how we think? Effects of priming individualism and collectivism. Psychological Bulletin, 134, 311-342. doi:10.1037/00332909.134.2.311

Panksepp, J. (1982). Toward a general psychobiological theory of emotions. The Behavioral and Brain Sciences, 5(3), 407-467.

Plutchik, R. (1991). The emotions. Lanham, MD: University Press of America.

Plutchik, R. (2002). Emotions and life: Perspectives from psychology, biology and evolution. Washington, DC: American Psychological Association.

Posner, J., Russell, J. A., \& Peterson, B. S. (2005). The circumplex model of affect: An integrative approach to affective neuroscience, cognitive development, and psychopathology. Development and Psychopathology, 17(3), 715-734. doi:10.1017/S0954579405050340

Reddy, W. M. (2008). The navigation of feeling. Cambridge, UK: Cambridge University Press. 
Russell, J. A. (1980). A circumplex model of affect. Journal of Personality and Social Psychology, 39(6), 1161-1178. doi:10.1037/h0077714

Russell, J. A. (1991). Culture and the categorization of emotions. Psychological Bulletin, 110(3), 426-450.

Russell, J. A. (2003). Core affect and the psychological construction of emotion. Psychological Review, 110(1), 145-72. doi:10.1037//0033-295X.110.1.145

Shaver, P., Schwartz, J., Kirson, D., \& O'Connor, C. (2001). Emotional knowledge: Further exploration of a prototype approach. In G. Parrott (Ed.), Emotions in social psychology: Essential readings (pp. 26-56). Philadelphia, PA: Psychology Press.

Shmelev, A. G. (2004). A test as a weapon. Psychology. Journal of Higher School of Economics, 1(2), 40-53. (in Russian)

Tulis, M., \& Fulmer, S. M. (2013). Students' motivational and emotional experiences and their relationship to persistence during academic challenge in mathematics and reading. Learning and Individual Differences, 27, 35-46. doi:10.1016/j.lindif.2013.06.003

Veirman, E., \& Fontaine, J. R. J. (2015). Revisiting the dimensional structure of the emotion domain. Cognition and Emotion, 29(6), 1026-1041. doi:10.1080/02699931.2014.963518

Watson, D., Clark, L. A., \& Tellegen, A. (1988). Development and validation of brief measures of positive and negative affect: the PANAS scales. Journal of Personality and Social Psychology, 54, 1063-1070.

Wessman, A. E., \& Ricks, D. F. (1966). Mood and personality. New York: Holt, Rinehart \& Winston.

Wilson-Mendenhall, C. D., Barrett, L. F., \& Barsalou, L. W. (2013). Neural evidence that human emotions share core affective properties. Psychological Science, 24(6), 947-956. doi:10.1177/ 0956797612464242

Yik, M., Russell, J. A., \& Steiger, J. H. (2011). A 12-point circumplex structure of core affect. Emotion, 11(4), 705-731. doi:10.1037/a0023980

Mikhail D. Malashenko - graduate, Vysokovsky Higher School of Urban Studies and Planning, National Research University Higher School of Economics.

E-mail: mihail_malashenko@mail.ru

Ekaterina A. Strizhova - associate professor, Psychoanalisis and business consulting department, School of Psychology, Faculty of Social Science, National Research University Higher School of Economics, Ph.D.

E-mail: estrizhova@hse.ru 